INPLASY

PROTOCOL

To cite: Qiu et al.

Comprehensive analysis of the safety of semaglutide in type 2 diabetes: a meta-analysis of the SUSTAIN and PIONEER trials. Inplasy protocol

2020110013. doi:

10.37766/inplasy2020.11.0013

Received: 04 November 2020

Published: 04 November 2020

Corresponding author:

Mei Qiu

13798214835@sina.cn

Author Affiliation:

Shenzhen Longhua District

Central Hospital

Support: None.

Review Stage at time of this submission: Preliminary

searches.

Conflicts of interest:

None.

\section{Comprehensive analysis of the safety of semaglutide in type 2 diabetes: a meta-analysis of the SUSTAIN and PIONEER trials}

Qiu, M1'; Ding, LL2; Zhou, HR³ .

Review question / Objective: A series of SUSTAIN and PIONEER trials have been completed and have validated the efficacy of semaglutide in patients with type 2 diabetes (T2D). However, none of them are powered to fully assess the safety of semaglutide.

Condition being studied: This meta-analysis will evaluate the risk of semaglutide in leading to various serious adverse events (SAEs) related to various systems of the human body. Information sources: We will search the SUSTAIN and PIONEER trials of semaglutide via two online databases (i.e., Embase and PubMed).

INPLASY registration number: This protocol was registered with the International Platform of Registered Systematic Review and Meta-Analysis Protocols (INPLASY) on 04 November 2020 and was last updated on 04 November 2020 (registration number INPLASY2020110013).

\section{INTRODUCTION}

Review question / Objective: A series of SUSTAIN and PIONEER trials have been completed and have validated the efficacy of semaglutide in patients with type 2 diabetes (T2D). However, none of them are powered to fully assess the safety of semaglutide.
Condition being studied: This metaanalysis will evaluate the risk of semaglutide in leading to various serious adverse events (SAEs) related to various systems of the human body.

\section{METHODS}

Participant or population: Adult individuals with T2D. 
Intervention: Semaglutide [a glucagon-like peptide-1 receptor agonist (GLP1RA)], used at different dosages via different routes of administration: subcutaneous semaglutide ( $0.5 \mathrm{mg} /$ week), subcutaneous semaglutide ( $1.0 \mathrm{mg} /$ week), oral semaglutide (3 mg/day), oral semaglutide ( $7 \mathrm{mg} / \mathrm{day})$, and oral semaglutide (14 $\mathrm{mg} /$ day).

Comparator: Non-semaglutide GLP1RA antihyperglycemic drug, non-GLP1RA antihyperglycemic drug, and placebo.

Study designs to be included: SUSTAIN trials and PIONEER trials assessing semaglutide in T2D patients.

Eligibility criteria: They are detailed in the above PICOS sections.

Information sources: We will search the SUSTAIN and PIONEER trials of semaglutide via two online databases (i.e., Embase and PubMed).

Main outcome(s): Various SAEs which were assessed in at least three of the SUSTAIN and PIONEER trials.

Data management: The articles identified by the retrieval of two online databases will be assessed for relevance according to their titles and abstracts, and then those studies potentially eligible will be assessed for the final eligibility according to the inclusion and exclusion criteria. Two authors will then independently extract the pre-specified data from the included studies using a standardized Excel data extraction sheet. The pre-specified data to be extracted contain study design, patient characteristics, type of intervention, type of control, the number of the occurrence of various SAEs of interest and that of participants. Any disagreements relevant with study selection and data extraction will be resolved through discussion with a third author. Treatment effects will be measured by pooled risk ratios (RRs) and 95\% confidence intervals (Cls) derived from meta-analysis of the dichotomous data in included studies.
Quality assessment / Risk of bias analysis: Two authors will use the Cochrane risk of bias assessment tool to independently assess the quality of included trials. Any disagreements related to quality assessment will be resolved through discussion with a third author.

Strategy of data synthesis: We will perform meta-analysis using the study-level binary data (i.e., the number of events in the intervention group, the number of patients in the intervention group, the number of events in the control group, and the number of patients in the control group). Drug effects will be presented as risk ratios (RRs) and 95\% confidence intervals (Cls). 12 statistic will be calculated to measure statistical heterogeneity. Cochran's $Q$ test will be used to test for treatment-bysubgroup interactions. Funnel plots and Egger tests will be done to assess the publication bias. All statistical analyses will be conducted in the Stata software (version 15.1).

Subgroup analysis: Subgroup analysis on all the endpoints of interest will be conducted according to different interventions [i.e., subcutaneous semaglutide ( $0.5 \mathrm{mg} /$ week), subcutaneous semaglutide $(1.0 \mathrm{mg} / \mathrm{week})$, oral semaglutide ( $3 \mathrm{mg} / \mathrm{day})$, oral semaglutide ( 7 $\mathrm{mg} /$ day), and oral semaglutide (14 $\mathrm{mg} /$ day)] and different comparators [i.e., nonsemaglutide GLP1RA antihyperglycemic drug, non-GLP1RA antihyperglycemic drug, and placebo].

Sensibility analysis: Both random-effects meta-analysis and fixed-effects metaanalysis will be conducted to assess the robustness of pooled results.

Country(ies) involved: China.

Keywords: type 2 diabetes, semaglutide, safety.

Contributions of each author:

Author 1 - Mei Qiu.

Author 2 - Liang-Liang Ding.

Author 3 - Hai-Rong Zhou. 